\title{
Investigaciones en rehabilitación cognitiva para pacientes con esquizofrenia (2004-2013): una revisión temática en beneficio de propuestas para etapas iniciales del trastorno Research in cognitive rehabilitation of patients with schizophrenia (2004-2013). A thematic review aimed at proposals for early stages of the disorder
}

Margarita Loubat O. ${ }^{1}$, Roberto Gárate M. ${ }^{2}$ y Nelson Cuturrufo A. ${ }^{3}$

\begin{abstract}
A thematic review of research in cognitive reabilitation of patients with schizophrenia is presented with the purpose of systematizing a contribution to the generation of intervention proposals for early stages of the disorder. Sudies are reviewed between 2004-2013 based on the "PubMed.gov" and "Scielo.org" search engines. Forty-two papers were found and 19 were selected, applying as inclusion criteria research with control groups that show positive results in the rehabilitation of attention, memory, and executive function. Furthermore, the aim is to get to know the cognitive rehabilitation program on which that research is based in order to appreciate its approach and its emphases. The results show a corpus of interventions which, in spite of their diversity, have a significant impact on the patients' cognitive and psychosocial functioning. The main predictors of good results are low age, low degree of cognitive deterioration, and low use of antipsychotic medication at the time of intervention. Improvement of executive functioning appears to be the best predictor of the patients' functionality in everyday activities. An impact is also seen of cognitive improvement on relevant clinical indicators: functioning in everyday activities, social adjustment, and clinical indices associated with a decrease of the observed symptoms. It is concluded that these results, together with research in which it is estimated that the alteration of the course of the schizophrenia requires early and multimodal work, imply the need to generate therapeutic proposals from the first episodes where the cognitive rehabilitation of the patient cannot be absent.
\end{abstract}

Key words: Schizophrenia, cognitive rehabilitation, first psychotic episodes.

Rev Chil Neuro-Psiquiat 2014; 52 (3): 213-227

Recibido: $19 / 05 / 2014$

Aceptado: 06/08/2014

Los autores no presentan ningún tipo de conflicto de interés.

Este estudio fue financiado por el proyecto DICYT No 030893LO, de la Dirección de Investigación Científica y Tecnológica de la Universidad de Santiago de Chile y realizado en colaboración con el Servicio de Psiquiatría del Hospital Clínico Dr. Félix Bulnes.

1 Psicóloga. Académica Escuela de Psicología USACH. Miembro de equipo de estudios Servicio de Psiquiatría Hospital Clínico Dr. Félix Búlnes.

2 Psicólogo, Profesor Escuela de Psicología USACH.

3 Médico Psiquiatra, Jefe Unidad Clínica Ambulatoria, Servicio de Psiquiatría Hospital Clínico Dr. Félix Bulnes. 


\section{Introducción}

$\mathrm{L}$ a esquizofrenia es un trastorno grave, estimándose una prevalencia de $0,5 \%$ en población mayor de 15 años y una incidencia en Chile de 12 casos nuevos por cada cien mil habitantes por año ${ }^{1}$. El inicio temprano y el índice de personas afectadas por alguna sintomatología del trastorno, la hacen ser unos de los problemas de salud que más contribuyen a la carga global de enfermedades a nivel país ${ }^{2}$, siendo esta patología responsable del 1,87\% del total de años de vida perdidos por muerte prematura y discapacidad en Chile ${ }^{3}$.

Respecto del déficit cognitivo que la patología conlleva, se estima que cerca del $85 \%$ de los pacientes experimenta algún grado de deterioro ${ }^{4}$, con rendimientos estimados entre 1 y 3 desviaciones estándar bajo el promedio de la población general, medición en base a batería de test agrupados por funciones.

Específicamente, el trastorno impacta la atención, memoria (fundamentalmente "working memory") y funcionamiento ejecutivo ${ }^{6-10}$, lo cual incide en el mal pronóstico de la enfermedad ${ }^{11,12}$ $y$ en el afrontamiento cotidiano interpersonal, dejando al sujeto más vulnerable al estrés social ${ }^{4}$.

Las alteraciones cognitivas están presentes incluso antes del primer diagnóstico, como parte del pródromo y éstas tienden a mejorar o mantenerse invariables al estabilizarse la situación clínica, o aumentar, con las recaídas y el paso de los años ${ }^{12-15}$.

No obstante, esta devastadora evidencia, existen estudios longitudinales que demuestran que la remisión de síntomas y la recuperación del paciente es posible bajo ciertas condiciones y sí se superan etapas $^{16,17}$. Ciertos autores ${ }^{18}$, señalan un paradigma de tres resultados terapéuticos jerarquizados: control de síntomas y prevención de recaídas; remisión mantenida de síntomas; y autonomía funcional y social del paciente. A estos antecedentes, se suma que existiría un período crítico y determinante para la evolución del trastorno, ubicado poco antes del primer episodio y 3 a 5 años posteriores al mis$\mathrm{mo}^{19}$; $\mathrm{y}$ que es en esa etapa del desarrollo del trastorno, donde se producirían cambios significativos a nivel neurobiológico como psicosocial, lo cual determinaría el curso futuro de la enfermedad ${ }^{20}$.
De lo señalado, se desprende la necesidad de realizar tratamientos integrales en etapas iniciales del trastorno, donde la rehabilitación cognitiva no debería estar ausente, considerando que el deterioro neuropsicológico puede incidir en el afrontamiento cotidiano interpersonal, dejando al sujeto más vulnerable al estrés social ${ }^{4}$; en el mal pronóstico de la enfermedad ${ }^{11}$, en la adherencia al tratamiento, la reinserción social y en la calidad de vida de los pacientes ${ }^{4}$.

Por lo tanto, se realiza una revisión de investigación considerando la rehabilitación de los dominios cognitivos: atención, memoria y función ejecutiva, que se sustentan en destacados programas de rehabilitación cognitiva para pacientes con esquizofrenia, con el objeto de sistematizar un corpus de intervenciones con resultados positivos y dar a conocer las técnicas utilizadas que han mostrado efectividad, a fin de aportar insumos que pudieran orientar intervenciones para pacientes que presentan primeros episodios psicóticos.

\section{Metodología}

Se revisan revistas Scopus, ISI, Scielo ("PubMed.gov" y "Scielo.org"), entre los años 20042013, asociando las palabras claves rehabilitación cognitiva, déficit cognitivos, esquizofrenia y psicosis. Se encuentran 42 artículos, los cuales se analizan en su totalidad. Se seleccionan 19, considerando como criterio de inclusión investigaciones que cuentan con grupo control y que muestran resultados positivos en rehabilitación de la atención, memoria y función ejecutiva. Se estima que si bien, ello, podría considerarse un "sesgo de publicación", el objetivo del trabajo lo hace ineludible. Adicionalmente, se busca conocer el programa de rehabilitación cognitiva, en el cual se basan dichas investigaciones, a fin de apreciar el acercamiento y sus énfasis.

\section{Resultados}

A continuación se describen los programas de rehabilitación utilizados y las investigaciones seleccionadas. 


\section{Integrated Psychological Therapy (IPT) for the Treatment of Neurocognition, Social Cognition and Social Competency in Schizophrenia, de Brenner et al.,1992}

Es un programa manual de terapia cognitivo conductual grupal. Se apoya en la premisa que los déficits cognitivos tienen un efecto preponderante sobre los niveles más altos de organización de la conducta: habilidades sociales o nivel de funcionamiento psicosocial ${ }^{21,22}$. Considera 5 subprogramas con niveles crecientes de complejidad asociados a neurocognición, cognición social, comunicación, habilidades sociales y habilidades de resolución de problemas interpersonales. Estos subprogramas pueden ser aplicados secuencialmente o por separado ${ }^{22}$.

Vita et al. ${ }^{23}$, compararon un grupo tratado con IPT y un grupo control. Reportaron que el grupo IPT mostró una mejoría significativamente mayor, respecto de variables psicopatológicas y de desempeño funcional, mejorando significativamente en memoria verbal, memoria de trabajo, con correlaciones especialmente significativas entre desempeño neurocognitivo y cambios a nivel de funcionamiento general.

Los mismos autores ${ }^{24}$, compararon el impacto de la intervención IPT, la intervención Computer Assisted Cognitive Remediation y un grupo control. Los resultados mostraron comparativamente al grupo control, una mejoría significativamente mayor para ambos grupos, en todas las variables cognitivas. Los dominios más responsivos fueron velocidad de procesamiento de información y memoria de trabajo. La efectividad de los dos métodos fue comparable. Se observó, adicionalmente, correlaciones entre funcionamiento cognitivo y nivel de desempeño funcional.

En un análisis posterior de las investigaciones señaladas $^{23,24}$, los autores reportaron que el tratamiento muestra mayor eficacia si el paciente cumple con las siguientes condiciones: menor uso de antipsicóticos, menor severidad de síntomas y mejor funcionamiento neurocognitivo de base (especialmente ejecutivo y memoria); además, la menor edad al momento de la intervención, como uno de los mejores predictores de mejoría a nivel de desempeño funcional ${ }^{25}$.

\section{Cognitive Enhancement Therapy (CET) de Hogarty \& Flesher, 199926}

Este programa opera en parejas y con software específicos, enfatiza el entrenamiento atencional y en estrategias de memoria. Está dirigido a la rehabilitación cognitiva en el contexto del tratamiento psiquiátrico convencional, focalizando esfuerzos en el impacto funcional de las alteraciones cognitivas y sociales desde una perspectiva pragmática y de mejoría adaptativa para la vida cotidiana. La propuesta incluye un acercamiento a las alteraciones cognitivas en términos de estilos cognitivos disfuncionales: empobrecidos (inhibición de los procesos de lenguaje, procesamiento, funcionamiento ejecutivo), desorganizados (disminución de la habilidad de control inhibitorio ejecutivo, desregulación afectiva y planificación, manifestándose formas de funcionamiento inefectivo y caótico) o rígidos (dificultad para desarrollar respuestas alternativas a problemas sociales ${ }^{26}$.

Hogarty et al. ${ }^{27}$, señalan, en los resultados de una intervención mediante CET, una mejoría del rendimiento cognitivo con indicadores asociados a un aumento en neurocognición, velocidad de procesamiento y rendimiento conductual (seguimiento a 12 meses); y, una mantención del aumento en neurocognición y velocidad de procesamiento, más el aumento en estilo cognitivo, cognición social y ajuste social (seguimiento a 24 meses).

En estudios posteriores, en base al programa $\mathrm{CET}^{28-30}$, se demostró el primer año una mejoría respecto a los déficits en estilo cognitivo disfuncional, cognición social, ajuste social y sintomatología psicótica. El segundo año se observó un fortalecimiento del rendimiento en los procesos antes mencionados con un efecto adicional sobreneurocognición y velocidad de procesamiento.

\section{Cognitive Remediation Therapy (CRT) de Wykes, Reeder, Corner, Williams y Everitt, $1999^{31}$}

Programa individual, orientado por una evaluación neuropsicológica inicial, a partir de la cual se desarrolla el plan de tratamiento. Consta de ejercicios de papel y lápiz, organizados desde niveles básicos hasta complejos, manejados en función del ritmo de avance del sujeto. 
Penadés et al. ${ }^{32}$, señalan que en el grupo de pacientes tratados con CRT, se observó una mejoría significativa en el rendimiento en memoria verbal, no verbal y funcionamiento ejecutivo; una disminución sobre la ansiedad y depresión de los pacientes; pero sólo un efecto indirecto e inespecífico sobre la memoria de trabajo, en un seguimiento de 6 meses. Agregan, que los pacientes intervenidos con CRT mostraron una mejoría significativa a nivel de funcionamiento social, demostrando con ello la incidencia de las variables cognitivas en el tratamiento general. Wylkes et al. ${ }^{33}$, comparan una intervención mediante CRT y un tratamiento de cuidado psiquiátrico convencional: el programa CRT produce una mejoría significativa en el rendimiento asociado a flexibilidad cognitiva y se observan efectos, en rango moderado, respecto a planeación. La mejoría en el rendimiento, tuvo un efecto directo adicional sobre el funcionamiento social, y en la disminución de la sintomatología general.

Penadés et al. ${ }^{34}$, compararon el impacto de la CRT y una intervención cognitivo conductual convencional, señalando un mayor impacto sobre la cognición y sobre el funcionamiento en el grupo tratado con CRT. Reportan, adicionalmente, el rol de la memoria verbal como el indicador más potente del nivel funcional de base. También que un mejoramiento del funcionamiento ejecutivo, es el mejor predictor del funcionamiento cotidiano de los pacientes.

\section{CTR. Modalidad Mental State Attribution Therapy (MSAT) y CTR. Modalidad Mental Flexibility Therapy (MFT) ${ }^{35}$}

MSAT: Programa de entrenamiento breve grupal basado en los conceptos de "estado mental", "atribución", "agencia y comunicación", y "contextos comunicativos". Esta terapia está centrada en mejorar la "atribución de estados mentales", a partir de ejercicios orientados a situaciones sociales.

MFT: Intervención grupal centrada en la remediación de la flexibilidad cognitiva con ejercicios orientados hacia situaciones sociales y actividades de la vida cotidiana.
Lecardeur et al. ${ }^{35}$, comparan el impacto de MSAT y MFT, e incluyen un grupo control. Reportan que no hubo abandonos; que las alteraciones cognitivas observadas disminuyeron en los grupos con aplicación de MSDT y MFT, no así en el grupo control. En la comparación entre modalidades, el grupo MFT mostró mejores resultados en la disminución subjetiva de los déficits cognitivos de parte de los pacientes.

\section{Thinking Skills for Work Program $(T S W P)^{36}$}

Programa de entrenamiento cognitivo en base a software para apoyar la reinserción laboral. El entrenamiento considera habilidades en atención, velocidad de procesamiento, memoria y aprendizaje, $y$ funciones ejecutivas.

Mcgurck et al..$^{37}$, demostraron que la intervención mediante TSWP asoció un mejor rendimiento con la reinserción laboral del grupo de pacientes tratados, durante el período de seguimiento (3 años). Los resultados señalan mayor número de sujetos empleados, mayor número de empleos, y mayor número de semanas y de horas trabajadas.

\section{Computer-assisted cognitive strategy training (CAST) de Vauth et al., 2005}

Programa computacional grupal, que considera estrategias de afrontamiento, centradas en los déficits de la atención, memoria verbal y planeación.

Vauth et $\mathrm{al}^{38}$, reportaron que los pacientes que recibieron CAST, más rehabilitación vocacional mostraron un mayor rendimiento asociado a atención y memoria verbal, pero no en planeación. Adicionalmente, se observó en los pacientes un mayor porcentaje de empleabilidad en un seguimiento de 12 meses. El análisis de regresión logística efectuado, demostró que una mejoría en el rendimiento en memoria de corto y largo plazo, permite predecir una más exitosa colocación laboral.

\section{Computer-assisted cognitive remediation (CACR) techniques}

CACR comprende un grupo de técnicas de entrenamiento a partir de software, capaces de 
tratar de manera selectiva diferentes dominios $\operatorname{cognitivos}^{39}$. Numerosos estudios han demostrado que CACR puede tener efectos sobre la sintomatología psicótica y el funcionamiento psicosocial, en pacientes con esquizofrenia ${ }^{40}$.

\section{CACR: Modalidad REHACOM (REHACOM ${ }^{\circledR}$ modules)}

Sistema de terapia mediante computador, para fortalecer el funcionamiento cognitivo, que ha mostrado eficiencia terapéutica en pacientes con daño cerebral adquirido. El sistema consiste en un programa básico y un número de procedimientos de entrenamiento, permitiendo al paciente progresar y recibir una retroalimentación respecto a su trabajo.

Según Cochet et al. ${ }^{41}$, las funciones atención/ concentración, memoria de trabajo, lógica y funciones ejecutivas, son las más relevantes para entrenar en base al programa.

En la investigación de d'Amato et al. ${ }^{42}$, el grupo tratado con REHACOM, mostró una mejoría cognitiva significativa respecto a atención/vigilancia, memoria de trabajo verbal, aprendizaje verbal, razonamiento y solución de problemas. No se observaron cambios significativos asociados a memoria de trabajo y aprendizaje no verbal, velocidad de procesamiento o medidas de desempeño funcional. En el grupo control, no hubo ninguna diferencia entre la medición pre y post tratamiento.

\section{CACR: Modalidad Cogpack (Marker Software $\left.{ }^{\circledR}\right)$ programme}

El programa Cogpack considera diferentes ejercicios neurocognitivos divididos en: Ejercicios de dominio específico, con entrenamiento de áreas cognitivas que se encuentran particularmente afectadas en la esquizofrenia (memoria y fluencia verbal, velocidad psicomotora y coordinación; funciones ejecutivas, memoria de trabajo y atención). Ejercicios de dominio no específico que requieren el uso de varias funciones, involucrando al mismo tiempo, cultura general, lenguaje y habilidades de cálculo. Gran parte de los ejercicios son flexibles y adaptables a distintos niveles de complejidad y el programa registra el desempeño del paciente en cada sesión, permitiéndole recibir retroalimentación y monitorear su progreso ${ }^{28}$.

Rauchensteiner et al. ${ }^{43}$, compararon el desempeño de pacientes de alto riesgo de psicosis y pacientes con esquizofrenia, tras un entrenamiento de 10 sesiones con Cogpack. Los resultados sólo señalaron una mejoría significativa asociada a atención y memoria en el grupo de pacientes de riesgo. Según los autores, este hallazgo pone de manifiesto la importancia de una intervención cognitiva temprana, a fin de maximizar el potencial terapéutico del entrenamiento neurocognitivo.

\section{Computerized working memory training program BRAINSTIM de Penner, Kobel y Opwis, 2006 ${ }^{44}$. Training Tool Brain Stim}

Programa computarizado para mejorar el desempeño de la memoria de trabajo. Está formado por tres módulos: dos centrados en aspectos espaciales de la memoria de trabajo, mientras el tercero se enfoca en aspectos verbales. El primer módulo, "mapa de la ciudad", está dirigido al entrenamiento de la orientación espacial, memorizando visual y/o verbalmente una ruta tipo. Con posterioridad, la ruta debe ser reconstruida de memoria en un mapa de la ciudad virtual. El segundo módulo, "formar parejas", está orientado a la memoria espacial. Los pacientes deben recordar la ubicación de dos cartas similares boca abajo. El último módulo "memorización de números", presenta una serie de números por una fracción breve de tiempo, serie que debe ser recuperada tras la resolución de una tarea aritmética de distracción; este módulo se centra en la memoria de trabajo verbal y el funcionamiento del "ejecutivo central", basado en el modelo de memoria de trabajo de Braddeley ${ }^{45,46}$. Todos los módulos se adaptan automáticamente a un nivel de dificultad acorde al nivel de desempeño del paciente. Luego de un número predeterminado de respuestas correctas, el módulo aumenta el grado de dificultad, mientras que una serie de respuestas incorrectas obligan al paciente a repetir el nivel previo. Con cada nivel exitosamente completado, aumenta la cantidad de información que debe ser memorizada.

Hubacher et al. ${ }^{47}$, comparó el rendimiento de 
dos grupos de pacientes, uno tratado con BRAINSTIM y un grupo control, reportando que la comparación en las medidas de funcionamiento cognitivo pre y post, muestra una mejoría en memoria de trabajo verbal y visual, y memoria visual de corto plazo, con un tamaño del efecto en rango de pequeño y extenso, en el grupo tratado con BRAINSTIM. En contraste, el grupo control mostró una disminución del rendimiento en memoria de trabajo verbal y solo cambios indirectos en memoria de trabajo visual y memoria de corto plazo visual.

\section{Brain fitness program (BFP)}

Este programa consiste en seis ejercicios computarizados diseñados para mejorar la velocidad y precisión del procesamiento de información auditiva. Los ejercicios se ajustan constantemente al nivel de dificultad apropiado al paciente con la finalidad de que le permitan mantener un rango constante de respuestas correctas (alrededor del $85 \%)$. Los intentos correctos son premiados con puntos y animaciones visuales.

El estudio de Murthy et al..$^{48}$, reportó que el entrenamiento con BFP mostró un significativo efecto en la habilidad para resolver las tareas de entrenamiento en el programa y hubo un aumento de la velocidad de procesamiento. Sin embargo, este efecto no se hizo extensivo al desempeño en evaluación neuropsicológica post intervención, donde no se encontraron cambios significativos. No se encontró relación entre los cambios en el desempeño en la tarea de entrenamiento y medidas cognitivas o funcionales post intervención.

\section{Problem Solving and Cognitive Flexibility training (REPYFLEC)}

Entrenamiento basado en estrategias para mejorar el funcionamiento ejecutivo y la metacognición. Se usa papel, lápiz y una pizarra (desarrollo de tareas, dar explicaciones y realizar ejemplos). De formato grupal (entre 4-6 personas) y los contenidos se dividen en dos áreas principales: resolución de problemas y flexibilidad cognitiva.

El estudio de Farreny et al..$^{49}$, comparó los resultados de una intervención mediante REPYFLEC y una intervención recreativa general. Los resultados mostraron que en el grupo de pacientes REPYFLEC se observó una mejora significativa en el desempeño ejecutivo, sintomatología negativa $y$ funcionamiento en el post tratamiento, comparados con el grupo control. En el seguimiento se observó una mantención importante del nivel ejecutivo y funcional post tratamiento.

\section{Metacognitive and social cognition training (MSCT)}

El MSCT, fue diseñado para trabajar sobre los errores atribucionales: extracción precipitada de conclusiones, sesgos atribucionales, sesgos contra evidencia contradictoria, y otros aspectos de la cognición social, tales como el reconocimiento de emociones, la teoría de la mente y la percepción social $^{50}$.

Rocha \& Queirós $\mathrm{s}^{50}$, compararon el programa, utilizando MSCT y un tratamiento psiquiátrico convencional. Los resultados mostraron en el grupo MSCT una mejoría significativa en teoría de la mente, percepción social, reconocimiento de emociones y funcionamiento social. Adicionalmente, se observó una reducción en el uso de sesgos atribucionales (saltos en proceso de evaluación de situaciones sociales), después del entrenamiento. No se observaron diferencias entre los grupos respecto de indicadores psicopatológicos generales.

\section{Social cognition and interaction training (SCIT)}

Intervención grupal basada en un programa manualizado, que comprende tres fases: 1) entrenamiento de reconocimiento de emociones, considerando el contexto social; 2) reconocimiento de estilos atribucionales y "comprensión de situaciones sociales"; $y, 3$ ) integración de estas habilidades a situaciones de la vida cotidiana ${ }^{51}$.

El estudio de Bartholomeusz et al. ${ }^{52}$, reportó que la intervención con SCIT mostró buena adherencia por parte de los pacientes, observándose una mejoría significativa en el reconocimiento emocional, funcionamiento social y desempeño funcional.

En las Tablas 1, 2, 3 se muestra una mirada panorámica y comparativa, considerando los re- 
sultados y las mediciones realizadas. Sólo la Tabla 3 está relacionada con rehabilitación cognitiva de primeros episodios psicóticos.

Todas las investigaciones tienen grupo control: control propiamente tal o control placebo. Además, los sujetos participantes fueron su propio control, mediante medidas pre y post intervención.

\section{Conclusiones}

Se constata que existe un escaso número de investigación respecto de la rehabilitación cognitiva para pacientes con esquizofrenia. Ello, a pesar de haber hecho una revisión de 10 años en revistas con impacto y de la importancia que reviste este tipo de intervención, para la autonomía funcional y social del paciente. Respecto de primeros episodios psicóticos se encontró sólo un estudio.

Las investigaciones analizadas muestran éxitos terapéuticos independientemente de la modalidad de la rehabilitación cognitiva-formatos grupales, individuales; entrenamiento mediante software o papel y lápiz; intervenciones multi-dominio y específicas-, dando cuenta de un impacto significativo en el funcionamiento cognitivo y/o psicosocial de los pacientes.

Los principales predictores de buenos resultados de intervenciones de rehabilitación cognitiva son: una menor edad, un menor grado de deterioro cognitivo y un menor uso de medicación antipsicótica al momento de la intervención. El mejoramiento del funcionamiento ejecutivo muestra ser el mejor predictor de la funcionalidad cotidiana de los pacientes. Se aprecia que las intervenciones inciden en variables psicopatológicas, de desempeño funcional y adaptación para la vida cotidiana en general; y que una mejoría significativa en la velocidad de procesamiento de la información, memoria de trabajo, cognición y ajuste social incidirían en la reinserción laboral. Lamentablemente, no todos los estudios cuentan con seguimiento para evitar la evanescencia, siendo ello una limitación pues se desconoce si la mejoría se sustenta en el tiempo. Nueve investigaciones expuestas no cuentan con seguimiento: 5 referidas a la rehabilitación en multidominios y 4 a dominios específicos, todo lo cual indudablemente induce sesgo en las conclusiones que se presentan.

No obstante, considerando los diversos programas de rehabilitación cognitiva, para pacientes con esquizofrenia, considerando los dominios cognitivos -atención, memoria y funcionamiento ejecutivo- pragmáticamente se señala: que ejercicios de diferenciación cognitiva, percepción social, comunicación verbal, resolución de problemas interpersonales, habilidades sociales, planeación y resolución de problemas, son significativos para incluir en las intervenciones. La utilización de situaciones sociales y actividades de la vida cotidiana se estiman beneficiosas, pues promueven el buen funcionamiento diario de los pacientes. Los ejercicios específicos para mejorar la atención, memoria visual, verbal, de trabajo, velocidad de procesamiento de la información, entre otros, a través de software u otro medio audio visual, también se estiman relevantes. Un monitoreo del progreso individual, considerando ritmo de avance y retroalimentación, se creen necesario independientemente de la modalidad de la intervención. Se agrega -considerando otros estudios que han mostrado evidencias- que las intervenciones deben ser progresivas y de complejidad ascendente, al igual que el ejercicio físico, considerando que el "entrenamiento cognitivo" produce cambios a nivel neurobiológico, a partir del aprendizaje realizado ${ }^{53}$.

Aparentemente los estudios más adecuados y replicables en etapas tempranas de la enfermedad, serían aquellos desarrollados a partir de los programas IPT, CET y CRT, ya que muestran, además de resultados significativos sobre el funcionamiento cognitivo, una estimación del impacto de la mejoría cognitiva sobre indicadores clínicos relevantes (funcionamiento psicosocial: funcionamiento en actividades cotidianas, ajuste social e índices clínicos asociados a una disminución de la sintomatología observada). El éxito de esos programas multidominio, parece indicar la importancia de un abordaje cognitivo multimodal que incluya un trabajo sobre varios procesos cognitivos (atencionales, memoria, diversos tipos de funciones ejecutivas) con material de trabajo multisensorial (visual, auditivo, táctil), beneficio dado por el uso del dispositivo tecnológico. 


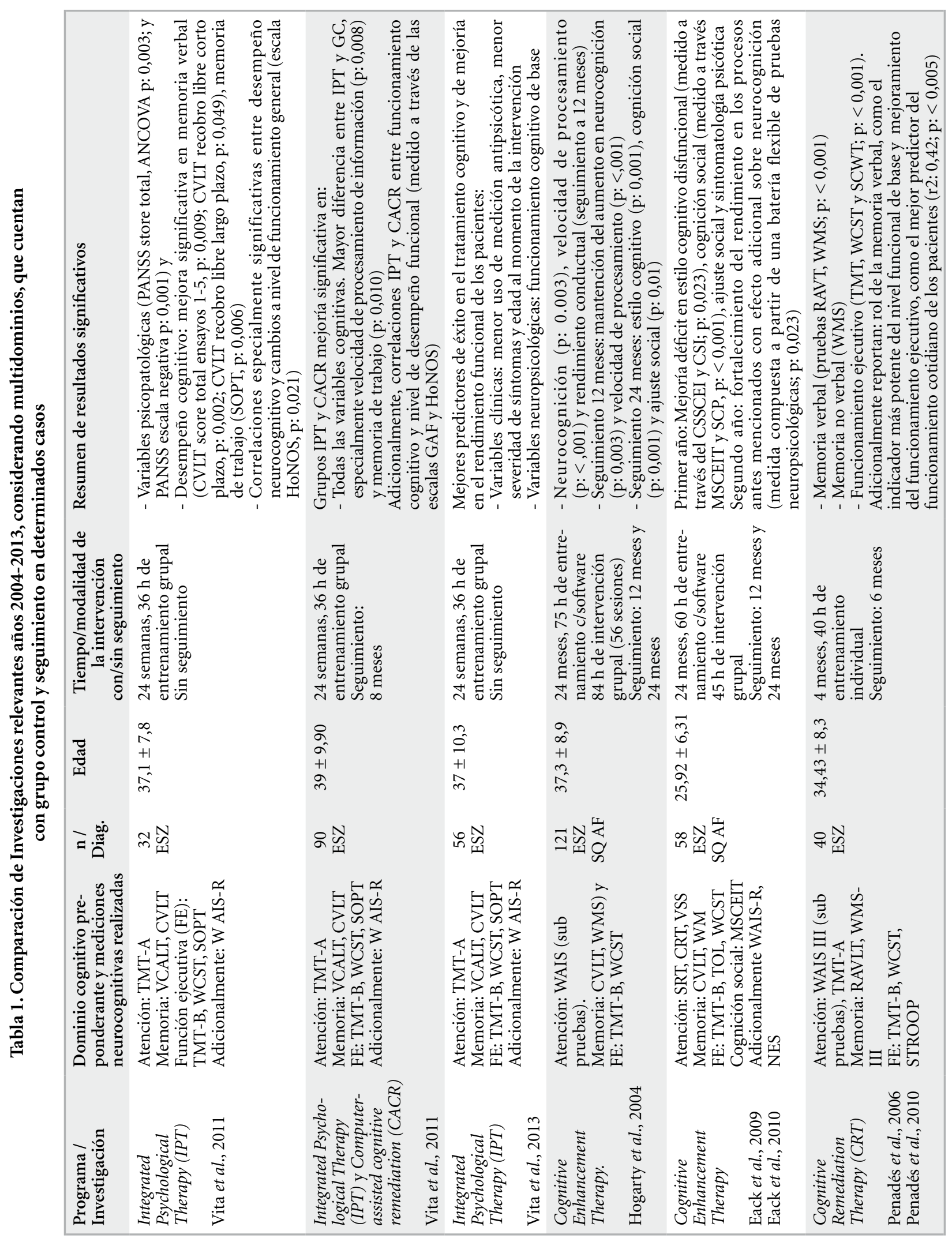




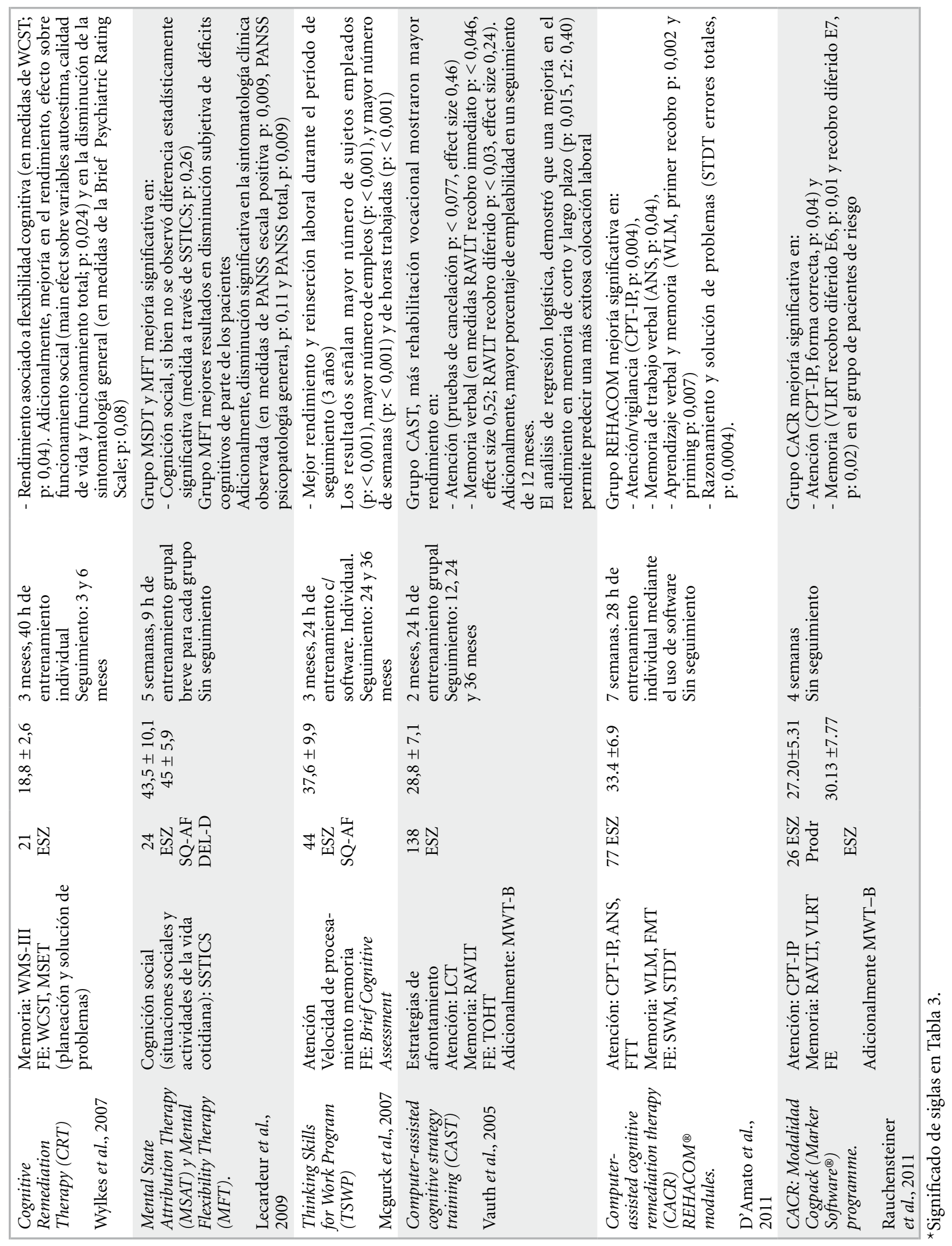




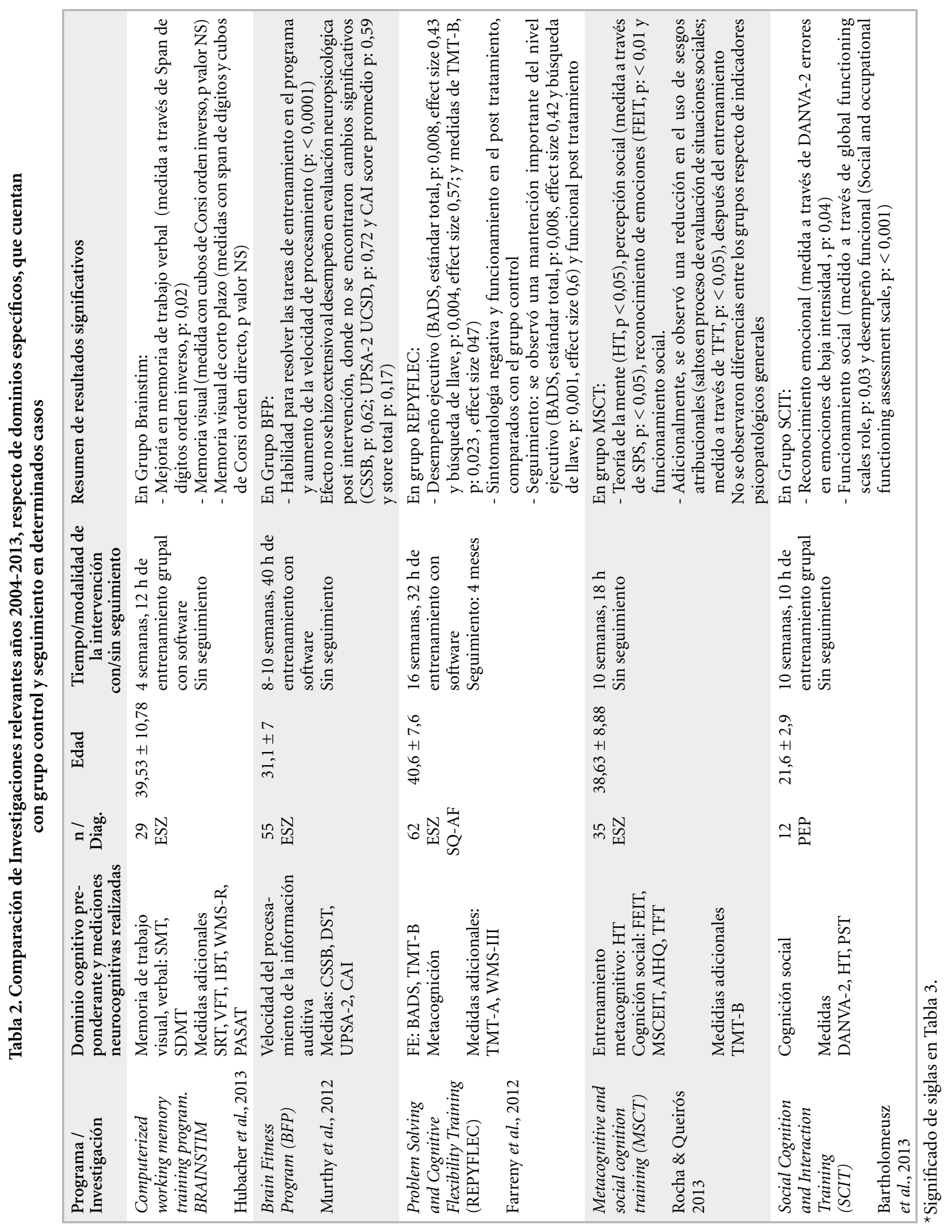


Tabla 3. Investigación relevante años 2004-2013, con pacientes primeros episodios psicóticos, respecto de dominios específicos y que cuenta con grupo control

\begin{tabular}{|c|c|c|c|c|c|}
\hline $\begin{array}{l}\text { Programa / } \\
\text { Investigación }\end{array}$ & $\begin{array}{l}\text { Dominio cognitivo } \\
\text { preponderante } \\
\text { y mediciones } \\
\text { neurocognitivas } \\
\text { realizadas }\end{array}$ & $\begin{array}{c}\text { n / } \\
\text { Diag. }\end{array}$ & Edad & $\begin{array}{l}\text { Tiempo/modalidad de } \\
\text { la intervención } \\
\text { con/sin seguimiento }\end{array}$ & Resumen de resultados significativos \\
\hline $\begin{array}{l}\text { Social Cognition } \\
\text { and Interaction } \\
\text { Training } \\
\text { (SCIT) } \\
\text { Bartholomeusz } \\
\text { et al., } 2013\end{array}$ & $\begin{array}{l}\text { Cognición social } \\
\text { Medidas } \\
\text { DANVA-2, } \\
\text { HT, PST }\end{array}$ & $\begin{array}{l}12 \\
\text { Primeros } \\
\text { Episodios } \\
\text { Psicóticos } \\
\text { (PEP) }\end{array}$ & $21,6 \pm 2,9$ & $\begin{array}{l}10 \text { semanas, } 10 \mathrm{~h} \text { de } \\
\text { entrenamiento grupal } \\
\text { Sin seguimiento }\end{array}$ & $\begin{array}{l}\text { Grupo SCIT mostró una mejoría } \\
\text { significativa en: } \\
\text { - Reconocimiento emocional (medida } \\
\text { a través de DANVA-2 errores en emo- } \\
\text { ciones de baja intensidad, p: } 0,04 \text { ) } \\
\text { - Funcionamiento social (medido a } \\
\text { través de global functioning scales } \\
\text { role, p: } 0,03 \text { y desempeño funcional } \\
\text { (Social and occupational functioning } \\
\text { assessment scale, p: }<0,001 \text { ) }\end{array}$ \\
\hline
\end{tabular}

*Siglas: 1BT 1-back task; AIHQ-A Ambiguous Intentions Hostility Questionnaire-ambiguous situations; ANS Auditory Number Sequencing; BADS Behavioral Assessment of the Dysexecutive Syndrome; CAI Cognitive Assessment Interview; CPT The Continuous Performance Test, CPT-IP The Continuous Performance Test-Identical Pair version; CRT choice reaction time; CSSB CogState ${ }^{\circledR}$ schizophrenia battery; CSSCEI Cognitive Style and Social Cognition Eligibility Interview; CSI Cognitive Styles Inventory; CT Cancellation task; CVLT California Verbal Learning Test; DANVA-2 Diagnostic Analysis of Non-verbal Accuracy-2; DL dichotic listening; DST Digit Span task; DT dual task; EAQ Everyday Attention Questionnaire; FEIT Facial Emotion Identification Test; FMT Face Memory Test; FTT Finger Tapping Test; HT Hinting task; LCT Letter cancellation test; MMSE Mini-Mental State Examination; MSCEIT Mayer-Salovey-Caruso Emotional IntelligenceTest; MSET Modified Six Elements Test; MWT-B Mehrfachwahl-Wortschatz-Intelligenztest; NES Neurological Evaluation Scale; PASAT Paced Auditory Serial Addition Task; PST Picture sequencing askRAVLT Rey Auditory Verbal Learning Test; SCP Social Cognition Profile; SCWT Stroop Color-Word Test; SDMT Symbol Digit Modalities Test; SMT selective reminding test; SOPT Self-Ordered Pointing Task; SPS Social Perception Scale; SRT Simple reaction time; SSTICS Subjective Scale to Investigate Cognition in Schizophrenia; STDT Strategic Target Detection test; SWM Spatial Working Memory test; TAVEC Spain-Complutense Verbal Learning Test; TFT the fish task; TMT-A Trail Making Test A; TMT-B Trail Making Test B; TOHT Tower of Hanoi Test; TOL Tower of London; UPSA-2 UCSD Performance based Skills Assessment; VCALT Visual Conditional Associative Learning Task; VFT verbal Fluency test; VLRT Verbal Learning and Retention Test; VSS visual-spatial scanning; WAIS-III; Wechsler Adult Intelligence Scale III v.; WAIS-R; Wechsler Adult Intelligence Scale R; WCST Wisconsin Card Sorting Test; WLM Word List Memory test; WMS Wechsler Memory Scale; WMS-R Wechsler Memory Scale revised. GAF Global Assessment of Functioning; HoNOS Health of the Nation Outcome Scales; PANSS Positive and Negative Syndrome Scale.

Es menester señalar que actualmente se han abierto nuevas áreas investigativas: Estudios respecto de la cognición social, especifican que si bien ésta requiere de capacidades cognitivas no-sociales (atención y memoria), cuando se estudia el impacto de ambas en el deterioro del funcionamiento diario de las pacientes, las capacidades cognitivas no sociales pierden fuerza explicativa, siendo el deterioro en la cognición social (por ejemplo en aspectos como teoría de la mente, incluso en el pródromo del trastorno), un elemento central en la explicación de esas deficiencias ${ }^{54-57}$. Estudios respecto del lenguaje, señalan alteraciones del lengua- je expresivo ${ }^{56}$ y pragmático, lo cual se traduciría en problemas cotidianos como expresar sentimientos, intereses y deseos ${ }^{57}$.

Estas nuevas áreas investigativas señalan que la cognición social sería mejor predictor de resultados funcionales, comparativamente a la función ejecutiva (resultados de la revisión temática aquí efectuada). Estas nuevas evidencias, indicarían la necesidad de matizar las intervenciones de rehabilitación cognitiva (sólo entrenamiento cognitivo), con aspectos del equilibrio del diario vivir, incluyendo tareas y ejercicios relacionados con problemas cotidianos interpersonales y de reinserción social-laboral. 
Por último, se estima que la revisión efectuada, promueve la incorporación de la rehabilitación cognitiva en el Programa Clínico Tratamiento de Personas desde Primer Episodio Esquizofrenia, Garantía Explícita de Salud (GES), de la Salud Pública chilena, a fin de impulsar la autonomía funcional y social del paciente y evitar una discapacidad secundaria: Las alteraciones y déficits cognitivos inciden en la adherencia al plan de tratamiento, en su calidad de vida, en el afrontamiento cotidiano interpersonal y funcionamiento diario de los pacientes.

\begin{abstract}
Resumen
Se presenta una revisión temática de investigaciones respecto de rehabilitación cognitiva para personas afectadas de esquizofrenia, con el propósito de hacer una sistematización que aporte a la generación de propuestas de intervención para etapas iniciales del trastorno. Se revisan estudios en base a los buscadores "PubMed.gov" y "Scielo.org", entre los años 2004-2013. Se encuentran 42 artículos y se seleccionan 19, considerando como criterio de inclusión investigaciones con grupo control y que muestran resultados positivos en rehabilitación de la atención, memoria y función ejecutiva. Adicionalmente, se busca conocer el programa de rehabilitación cognitiva, en el cual se basan dichas investigaciones, a fin de apreciar el acercamiento y sus énfasis. Los resultados dan cuenta de un corpus de intervenciones que, a pesar de su diversidad, impactan significativamente en el funcionamiento cognitivo y psicosocial de los pacientes. Los principales predictores de buenos resultados son: una menor edad, un menor grado de deterioro cognitivo y un menor uso de medicación antipsicótica al momento de la intervención. El mejoramiento del funcionamiento ejecutivo muestra ser el mejor predictor de la funcionalidad cotidiana de los pacientes. También, se aprecia un impacto de la mejoría cognitiva sobre indicadores clínicos relevantes: funcionamiento en actividades cotidianas, ajuste social e indices clinicos asociados a una disminución de la sintomatología observada. Se concluye que estos resultados, sumados a investigaciones donde se estima que la alteración del curso de la esquizofrenia requiere un trabajo precoz y multimodal, implican la necesidad de generar propuestas terapéuticas desde los primeros episodios donde la rehabilitación cognitiva del paciente no puede estar ausente.
\end{abstract}

Palabras claves: Esquizofrenia, rehabilitación cognitiva, primeros episodios psicóticos.

\section{Referencias bibliográficas}

1. Ministerio de Salud de Chile [MINSAL]. Guía Clínica Primer Episodio Esquizofrenia. SantiagoChile: MINSAL: 2005.

2. Ministerio de Salud de Chile [MINSAL]. Tratamiento de Personas desde Primer Episodio Esquizofrenia, Garantía Explícita de Salud (GES). Santiago-Chile: MINSAL: 2009.

3. Ministerio de Salud de Chile [MINSAL]. Estudio de Carga de Enfermedad. Santiago-Chile: MINSAL: 1996.

4. Cavieres A, Valdebenito M. Funcionamiento cog- nitivo y calidad de vida en la esquizofrenia. Rev Chil Neuro-Psiquiat 2005; 43: 97-108.

5. Saykin A, Gur RC, Gur RE, Mozley D, Mozley L, Resnick S, et al. Neuropsychological Function in Schizophrenia. Arch Gen Psychiatry 1991; 48: 61824.

6. Cuesta MJ, Peralta V, Zarzuela A. Neuropsicología y esquizofrenia. Anales del Sistema Sanitario de Navarra 2000; 23: 51-62.

7. Gold J. Cognitive deficits as treatment targets in schizophrenia. Schizophrenia Research 2004; 72: 21-8.

8. Kurtz MM. Neurocognitive impairment across the 
life span in schizophrenia: an update. Schizophr Research 2005; 74: 15-26.

9. Barrera A. Los trastornos cognitivos de la esquizofrenia. Rev Chil Neuro-Psiquiat 2006; 44 (3): 215-21.

10. De la Higuera J, Sagastagoitia E. Rehabilitación Cognitiva en la Esquizofrenia: Estado actual y perspectivas futuras. Revista Apuntes de Psicología 2006; 24 (1-3): 245-66.

11. Cano JF, Fierro-Urresta M, Vanegas CR, Alzate M, Olarte A, Cendales R, et al. Factores Pronósticos de Esquizofrenia en Primer Episodio Psicótico. Revista Salud Pública 2007; 9 (3): 455-64.

12. Palma C, Cañete J, Farriols N, Soler F, Julià, J. Primeros episodios psicóticos: características clínicas y patrones de consumo de sustancias en pacientes ingresados en una unidad de agudos. Revista Española Anales de Psicología 2005; 21 (2): 286-93.

13. Ojeda N, Sánchez T, Elizagárate E, Llölled A, Ezcurra J, Ramírez I, et al. Evolución de los síntomas cognitivos. Actas Españolas de Psiquiatría 2007; 35 (4): 263-70.

14. Haro JM, Ciudad A, Alonso J, Bousoño M, Suárez $\mathrm{D}$, Novick D, et al. Remisión y recaída en el tratamiento ambulatorio de los pacientes con esquizofrenia. Resultados a 3 años. Actas Españolas de Psiquiatría 2008; 36 (4): 187-96.

15. Elsawy H, El-Hay MA, Badawy A. CognitiveFunctions in First Episode Psychosis. Current Psychiatry 2010; 17 (4): 21-7.

16. Haro J, Edgell E, Jones P, Alonso J, Gavart S, Gregor K, et al. The European Schizophrenia Outpatient Health Outcomes (SOHO) study: rationale, methods and recruitment. Acta Psychiatrica Scandinavica 2003; 107 (3): 222-32.

17. San L, Ciudad A, Álvarez E, Bobes J, Gilaberte I. Symtomatic remission and social/vocational functioning in outpatiens with aschizophrenia: prevalenceand associations in a cross-sectional study. European Psychiatry 2007; 22 (8): 490-8.

18. Ciudad A, Bobes J, Álvarez E, San L, Novick D, Gilaberte I. Resultados clínicos relevantes en esquizofrenia: remisión y recuperación. Revista de Psiquiatría y Salud Mental 2011; 4 (1): 53-65.

19. Birchwood M, Todd P, Jackson C. Early intervention in psychosis. The critical period hypothesis.
British Journal of Psychiatry 1998; 172 (33): 53-9.

20. MacGorry P, Killackey E, Yung A. Early intervention in psychotic disorders: detection and treatment of the first episode and the critical early stages. Medical Journal of Australia 2007; 187 (7): 8-10.

21. Brenner H, Hodel B, Roder V, Corrigan, P. Treatment of cognitive dysfunctions and behavioral deficits in schizophrenia. Schizophrenia Bulletin 1992; 18 (1): 21-6.

22. Roder V, Mueller D, Schmidt S. Effectiveness of Integrated Psychological Therapy (IPT) for Schizophrenia Patients: A Research Update. Schizophr Bull 2011; 37 (Suppl 2): 71-9.

23. Vita A, De Peri L, Barlati S, Cacciani P, Cisima, M, Deste G, et al. Psychopathologic, neuropsychological and functional outcome measures during cognitive rehabilitation in schizophrenia: A prospective controlled study in a real-world setting. European Psychiatry 2011; 26: 276-83.

24. Vita A, Deste G, De Peri L, Barlati S, Poli R, Cesana $B$, et al. Predictors of cognitive and functional improvement and normalization after cognitive remediation in patients with schizophrenia. Schizophrenia Research 2013; 150. doi:10.1016/j. schres.2013.08.011.

25. Vita A, De Peri L, Barlati S, Cacciani P, Deste G, Poli R, et al. Effectiveness of different modalities of cognitive remediation on symptomatological, neuropsychological, and functional outcome domains in schizophrenia: a prospective study in a real-world setting. Schizophrenia Research 2011; 133 (1-3): 223-31.

26. Hogarty G, Flesher S. Practice principles of cognitive enhancement therapy for schizophrenia. Schizophrenia Bulletin 1999; 25 (4): 693-708.

27. Hogarty G, Flesher S, Ulrich R, Carter M, Greenwald $D$, Pogue-Geile $M$, et al. Cognitive enhancement therapy for schizophrenia: effects of a 2-year randomized trial on cognition and behavior. Archives of General Psychiatry 2004; 61: 866-76.

28. Eack S, Greenwald D, Hogarty S, Cooley S, DiBarry A, Montrose D, et al. Cognitive Enhancement Therapy for Early-Course Schizophrenia: Effects of a Two-Year Randomized Controlled Trial. Psychiatric Services 2009; 60 (11): 1468-76. 
29. Eack S, Greenwald D, Hogarty S, Keshavan M. One-year durability of the effects of cognitive enhancement therapy on functional outcome in early schizophrenia. Schizophrenia Research 2010; 120: 110-216.

30. Eack S, Hogarty G, Cho R, Prasad K, Greenwald D, Hogarty S, et al. Neuroprotective effects of cognitive enhancement therapy against gray matter loss in early schizophrenia. Arch Gen Psychiatry 2010; 67 (7): 674-82.

31. Wykes T, Reeder C, Corner J, Williams C, Everitt B. The effects of neurocognitive remediation on executive processing in patients with schizophrenia. Schizophrenia Bulletin 1999; 25 (2): 291-307.

32. Penadés R, Catalán R, Salamero Bogeta T, Puig O, Guarcha J, Gastó C. Cognitive Remediation Therapy for outpatients with chronic schizophrenia: A controlled and randomized study. Schizophrenia Research 2006; 87 (1-3): 323-31.

33. Wykes T, Newton E, Landau S, Rice Chr, Tompson N, Frangou S. Cognitive remediaton therapy (CRT) four young early onset patients with achizophrenia: An exploratory randomized controlled trial. Schizophrenia Research 2007; 94: 221-30.

34. Penadés R, Catalán R, Puig O, Masana G, Pujol $\mathrm{N}$, Navarro V, et al. Executive function needs to be targeted to improve social functioning with Cognitive Remediation Therapy (CRT) in schizophrenia. Psychiatry Research 2010; 177: 41-5.

35. Lecardeur L, Stip E, Giguere M, Blouin G, Rodríguez J, Champagne-Lavaua $M$. Effects of cognitive remediation therapies on psychotic symptoms and cognitive complaints in patients with schizophrenia and related disorders: A randomized study. Schizophrenia Research 2009; 111 (1-3): 153-8.

36. McGurk SR, Mueser KT, Pascaris A. Cognitive training and supported employment for persons with severe mental illness: one-year results from a randomized controlled trial. Schizophr Bull 2005; 31 (4): 898-909.

37. McGurk S, Mueser K, Feldman K, Wolfe R, Pascaris A. Cognitive Training for Supported Employment: 2-3 Year Outcomes of a Randomized Controlled Trial. American Journal of Psychiatry. 2007; 164 (3): 437-41.
38. Vauth R, Corrigan P, Clauss M, Dietl M, DreherRudolph M, Rolf-Dieter S, et al. Cognitive Strategies Versus Self-Management Skills as Adjunct to Vocational Rehabilitation. Schizophrenia Bulletin 2005; 31 (1): 55-66.

39. Grynszpan O, Perbal S, Pelissolo A, Fossati P, Jouvent R, Dubal S, et al. Efficacy and specificity of computer-assisted cognitive remediation in schizophrenia: a meta-analytical study. Psychological Medicine 2010; 41 (1): 163-73.

40. Wexler B, Bell M. Cognitive remediation and vocational rehabilitation for schizophrenia. Schizophrenia Bulletin 2005; 31 (4): 931-41.

41. Cochet A, Saoud M, Gabriele S, Broallier V, El Asmar C, Daléry J, et al. Impact of a new cognitive remediation strategy on interpersonal problem solving skills and social autonomy in schizophrenia. Encephale 2006; 32: 189-95.

42. D'Amato T, Bationa R, Cochetc A, Jalenquesd I, Gallandd F, Giraud-Barog E, et al. A randomized, controlled trial of computer-assisted cognitive remediation for schizophrenia. Schizophrenia Research 2011; 125 (2-3): 284-90.

43. Rauchensteinera S, Kawohlb W, Ozgurdalc S, Littmanna E, Gudlowskia Y, Witthausa H, et al. Test-performance after cognitive training in persons at risk mental state of schizophrenia and patients with schizophrenia. Psychiatry Research 2011; 185 (3): 334-9.

44. Penner I, Kobel M, Opwis K. BrainStim-a recently developed tool to train diferent aspects of working memory. Proceedings of the INS/GNP Conference 2006; 17-9.

45. Baddeley AD. Working Memory. Science New Series 1992; 255 (5044): 556-9.

46. Baddeley AD. The episodic buffer: a new component of working memory? Trends Cogn Sci 2000; 4: 417-23.

47. Hubacher M, Weiland M, Calabrese P, Stoppe G, Stöcklin M, Fischer-Barnicol D, et al. Working Memory Training in Patients with Chronic Schizophrenia: A Pilot Study. Psychiatry Journal 2013. doi:10.1155/2013/154867.

48. Murthy N, Mahncke H, Wexler B, Maruff P, Inamdar A, Zucchetto M, et al. Computerized cognitive remediation training for schizophrenia: An 
open label, multi-site, multinational methodology study. Schizophrenia Research 2012; 139: 87-91.

49. Farreny A, Aguado J, Ochoa S, Huerta-Ramos E, Marsà F, López-Carrilero R, et al. REPYFLEC cognitive remediation group training in schizophrenia. Looking for an integrative approach. Schizophrenia Research 2012; 142: 137-44.

50. Rocha N, Queirós C. Metacognitive and social cognition training (MSCT) in schizophrenia: A preliminary efficacy study. Schizophrenia Research 2013; 150: 64-8.

51. Penn D, Roberts D, Combs D, Sterne A. Best practices: the development of the Social Cognition and Interaction Training program for schizophrenia spectrum disorders. Psychiatric Services 2007; 58: 449-51.

52. Bartholomeusz C, Allott K, Killackey E, Liu P, Wood S, Thompson A. Social cognition training as an intervention for improving functional outcome in first-episode psychosis: a feasibility study. Early Intervention in Psychiatry 2013; 7:
421-6.

53. Genevsky A, Garrett C, Alexander P, Vinogradov S. Cognitive training in schizophrenia: a neuroscience-based approach. Dialogues in Clinical Neuroscience 2010; 12 (3): 416-21

54. Billeke P, Aboitiz F. Social cognition in schizophrenia: from social stimuliprocessingto social engagement. Front Psychiatry 2013; 4: 1-12.

55. Stilo S, Di Forti M, Mondelli V, Falcone AM, Russo M, O’Connor J, et al. Social Disadvantage: Cause or Consequence of Impending Psychosis? Schizophrenia Bulletin 2013; 39 (6): 1288-95.

56. Tavano A, Sponda S, Fabbro F, Perlini C, Rambaldelli G, Ferro A, et al. Specific linguistic and pragmatic deficits in Italian patients with schizophrenia. Schizophrenia Research 2008; 102: 53-62.

57. Durán E, Figueroa A. Sobre el déficit pragmático en la utilización de pares adyacentes, por pacientes esquizofrénicos crónicos, y de primer brote. Rev Chil Neuro-Psiquiat 2009; 47 (4): 259-70.
Correspondencia:

Psicóloga Margarita Loubat O.

Escuela de Psicología. Universidad de Santiago de Chile.

Avda. Ecuador 3650, Estación Central, Santiago

de Chile.

E-mail :margarita.loubat@usach.cl 